\title{
Kinematic Alignment in Cruciate Retaining Implants Improves the Biomechanical Function in Total Knee Arthroplasty during Gait and Deep Knee Bend
}

\author{
Kyoung-Tak Kang, $\mathrm{PhD}^{1, *}$ Yong-Gon Koh, MD², Ji Hoon Nam, MS² Sae Kwang Kwon, MD² \\ Kwan Kyu Park, MD, PhD 3 \\ ${ }^{1}$ Department of Mechanical Engineering, Yonsei University, \\ Seodaemun-gu, Seoul, Republic of Korea \\ ${ }^{2}$ Department of Orthopaedic Surgery, Joint Reconstruction Center, \\ Yonsei Sarang Hospital, Seocho-gu, Seoul, Republic of Korea \\ ${ }^{3}$ Department of Orthopedic Surgery, Yonsei University College of \\ Address for correspondence Kwan Kyu Park, MD, PhD, Department of \\ Orthopedic Surgery, Yonsei University College of Medicine, 50-1 \\ Yonsei-ro, Seodaemun-gu, Seoul, 03722, Republic of Korea \\ (e-mail: KKPARK@yuhs.ac).
} Medicine, Seodaemun-gu, Seoul, Republic of Korea

J Knee Surg 2020;33:284-293.

\begin{abstract}
Kinematic alignment (KA), which co-aligns the rotational axes of the components with three kinematic axes of the knee by aligning the components to the prearthritic joint lines, has been a recently introduced surgical technique. However, whether KA and cruciate retaining (CR) implants provide better biomechanical function during activities than mechanical alignment (MA) in posterior stabilized (PS) implants is unclear. We evaluated the biomechanical functions during the stance phase gait and deep knee bend, with a computer simulation and measured forces in the medial and lateral collateral ligaments and medial and lateral contact stresses in the polyethylene insert and patellar button. The forces on the medial collateral ligament in KA were lower than those in MA in both CR and PS TKA in the stance

Keywords

- total knee arthroplasty

- kinematic alignment

- mechanical alignment

- finite element analysis phase gait and deep knee bend conditions, whereas those on the lateral collateral ligament did not show any difference between the two surgical alignment techniques in the stance phase gait condition. The maximum contact stresses on the medial PE inserts in KA were lower than those in MA in both CR and PS TKA in the stance phase gait and deep knee bend conditions. However, the maximum contact stresses on the lateral PE inserts and the patellar button did not differ between MA and KA. The biomechanical function was superior in KA TKA than in MATKA, and KA was more effective in CR TKA. This comparison could be used as a reference by surgeons to reduce the failure rates by using KA TKA instead of MA TKA.
\end{abstract}

For both surgeons and patients, the effects of alignment on the lifespan and postoperative function of prostheses are significant factors in total knee arthroplasty (TKA). ${ }^{1-3}$ Proper restoration of knee alignment reduces both the mechanical and shear stresses exerted on the polyethylene (PE) insert as well as on the interfaces between the bone and the prosthe-

Kyoung-Tak Kang and Young-Gon Koh contributed equally to this work and should be considered co-first authors.

received

January 15, 2018

accepted after revision

December 16, 2018

published online

February 6, 2019 sis. $^{4,5}$ Several surgical techniques with functional or anatomical reference axes have been developed and introduced to achieve this goal. ${ }^{6}$

A successful outcome is believed to be dependent on the restoration of a neutral mechanical axis in the lower extremity, with the femoral and tibial components aligned perpendicular to the mechanical axis in the coronal plane. Although mechanical alignment (MA) is the most common surgical technique in TKA for restoring patient function, 15 to $25 \%$ of the patients

Copyright $\odot 2020$ by Thieme Medical Publishers, Inc., 333 Seventh Avenue, New York, NY 10001, USA.

DOI https://doi.org/ $10.1055 / \mathrm{s}-0039-1677846$ ISSN 1538-8506. 
have reported dissatisfaction, and $10 \%$ required revision surgery within 10 years of the surgery. ${ }^{7-10}$ Recently, it has been suggested that MA may not be the best solution for overall component survivorship and clinical function. ${ }^{11-13}$

Kinematic alignment (KA) is a recent modification in surgical technique that suggests aligning the angle, level, and posterior joint line of the femoral component and the joint line of the tibial component to those of the prearthritic normal knee joint. ${ }^{14}$ In previous studies, it was found that patients undergoing surgery with the KA TKA method reported better relief, function, and flexion than did patients with MA TKA. ${ }^{15,16}$ In KA TKA, the reference line for the internal-external rotation of the anterior-posterior axis of the tibial component is the flexion-extension tibial reference line, which is a functionally-not anatomically defined reference line, with an orientation parallel to the flexionextension plane of the extended knee after compensating for wear which is primarily cartilage without connecting anatomic landmarks on the tibia. ${ }^{17-19} \mathrm{KA}$ corrects the arthritic deformity to the constitutional alignment of the patient, with restorations of the femoral and tibial components to the natural tibiofemoral articular surface, alignment, and natural laxities of the knee. ${ }^{15,19}$ However, joint-line preservation with those of the normal knee also leads to the tibial component, in which posterior wear or loosening from inadvertent placement of the tibial component could lead to early failure or revision surgery. ${ }^{3}$ Furthermore, the incidence of failure is three to five times lower in KA TKA than in MA TKA. ${ }^{3,20,21}$ In addition, Nedopil et al showed that incidence of tibial component failure after KA TKA was $0.3 \%$ and was caused not by varus subsidence, but by posterior subsidence or posterior edge wear. ${ }^{20}$ They demonstrated that the strategy for lowering the risk of tibial component failure when performing KA is to set the tibial component parallel to the flexion-extension plane and varus-valgus plane of the native joint line. ${ }^{20}$ However, the biomechanical effects of MA TKA and KA TKA have not been reported.

Therefore, the present study used finite element (FE) analysis during both stance phase gait and deep knee bend to determine whether the use of KA or MA, or the use of cruciate retaining (CR) and posterior stabilized (PS) TKA resulted in lower forces in the medial and lateral collateral ligaments (MCLs and LCLs), lower maximum contact stress in the medial and lateral compartment of the tibial insert, and lower maximum contact stresses in the patellar button.

\section{Materials and Methods}

\section{Development of FE Model}

We used a three-dimensional nonlinear FE model for the knee joint developed using the computed tomography (CT) and magnetic resonance imagings (MRIs) of a healthy 36year-old male subject. ${ }^{22,23}$ The computational knee-joint model was developed on the basis of bony structures, including the femur, tibia, fibula, and patella, and soft tissues, such as ligaments and menisci, reconstructed from the CT and MRIs, respectively-a process that has been established and validated in previous studies. ${ }^{22,23}$
Bones are much stiffer than the soft tissue and were assumed to be rigid, and all major ligaments were modeled using nonlinear and tension-only spring elements. ${ }^{23-26}$ The force-displacement relationship based on the functional bundles in the actual ligament anatomy refers to the following ${ }^{27}$ :

$$
f(\varepsilon)=\left\{\begin{aligned}
\frac{k \varepsilon^{2}}{4 \varepsilon_{1}}, & 0 \leq \varepsilon \leq 2 \varepsilon_{1} \\
k\left(\varepsilon-\varepsilon_{1}\right), & \varepsilon>2 \varepsilon_{1} \\
0, & \\
\varepsilon & =\frac{l-l_{0}}{l_{0}} \\
l_{0} & =\frac{l_{r}}{\varepsilon_{r}+1}
\end{aligned}\right.
$$

where $f(\varepsilon)$ is the current force, $k$ is the stiffness, $\varepsilon$ is the strain, and $\varepsilon_{1}$ is assumed to be constant at 0.03 . The ligament bundle slack length 10 can be calculated by using the reference bundle length $l_{r}$ and the reference strain $\varepsilon_{r}$ in the upright reference position. The PE insert and patellar button were modeled as an elastoplastic material (-Table 1). ${ }^{23}$ All implant components were modeled as linear elastic isotropic materials (- Table $\mathbf{1}$ ). ${ }^{23}$

Contact conditions were applied between the femoral component, PE insert, and patellar button in TKA. The coefficient of friction between the PE material and metal was chosen to be 0.04 for consistency with previous explicit FE models. ${ }^{23,28}$ Contact was defined by using a penalty-based method with a weighting factor. As a result, the contact forces were defined as a function of the penetration distance of the master into the slave surface. The femoral and tibial components were fully bonded to the femur and tibia bone models, respectively.

\section{FE Models for MA and KA}

To develop the FE models for MA TKA and KA TKA, two experienced surgeons (the third and last authors) performed surgical simulations for both surgical technologies. We developed the FE model for MA TKA according to the following surgical preferences: (1) the default alignment for the femoral component rotation was parallel to the transepicondylar axis with the coronal alignment perpendicular to the mechanical axis with a $9.5 \mathrm{~mm}$ distal medial resection, and (2) the sagittal alignment was at a $3^{\circ}$ flexion. The tibial default alignment was rotated $0^{\circ}$ to the anterior-posterior axis, the coronal alignment was $90^{\circ}$ to the mechanical axis, and the sagittal alignment was $5^{\circ}$ of the posterior to the mechanical axis. The method for aligning the components for KA was developed by Howell et al. ${ }^{14,19}$ We placed the femoral component at the angle and level of the distal and posterior joint line of the distal femur first, and the tibial component was placed to restore the natural

Table 1 Material properties for finite element model

\begin{tabular}{|l|l|l|}
\hline & Young's modulus (MPa) & Poisson's ratio \\
\hline CoCrMo alloy & 220,000 & 0.30 \\
\hline UHMWPE & 685 & 0.47 \\
\hline Ti6Al4V alloy & 110,000 & 0.30 \\
\hline
\end{tabular}




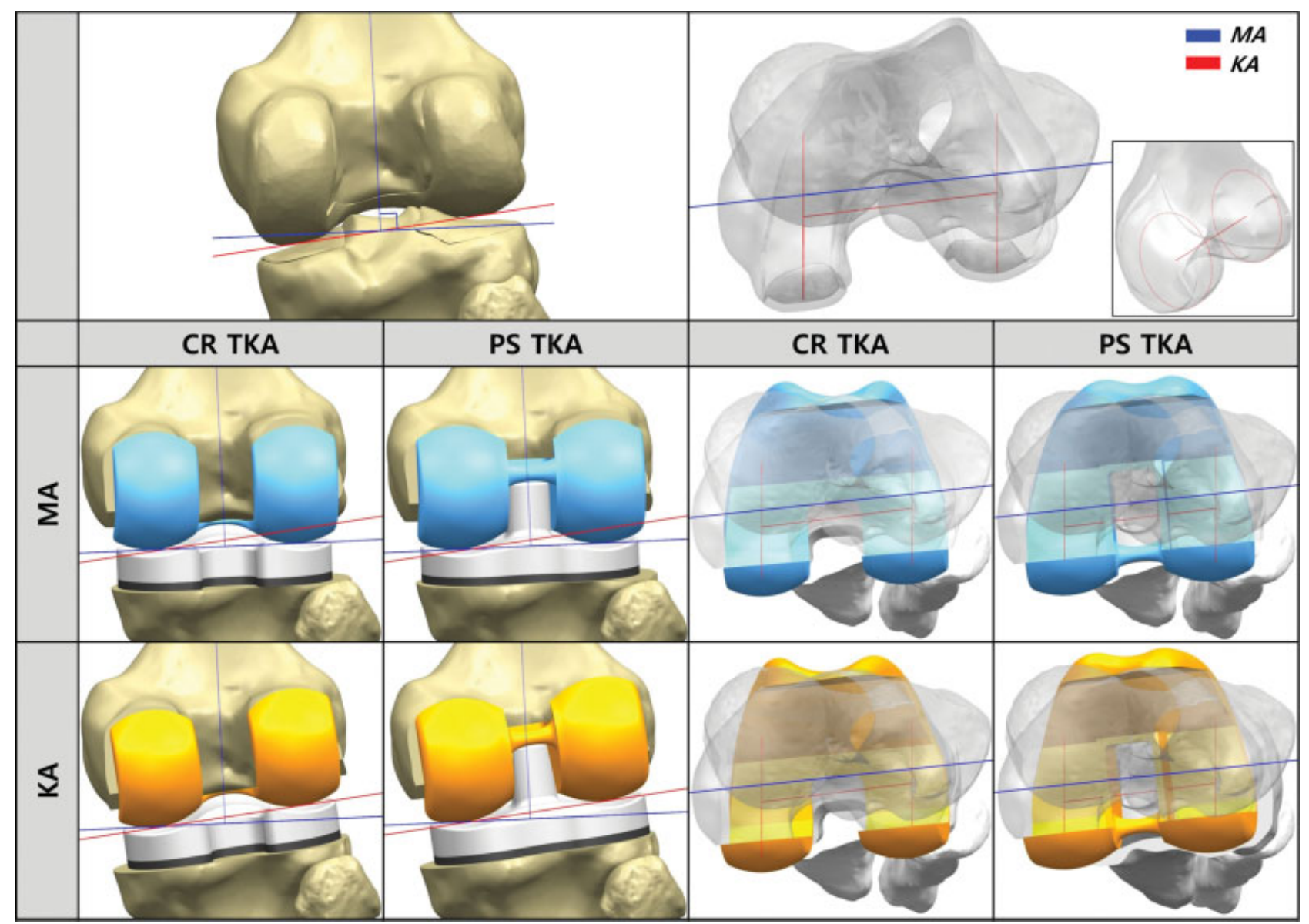

Fig. 1 Finite element models used in analysis for mechanical alignment (MA) total knee arthroplasty (TKA) and kinematic alignment (KA) TKA models in cruciate retaining (CR) and posterior stabilized (PS) TKA.

proximal distal-level posterior slope and varus-valgus angulation of the tibial joint line. ${ }^{21}$ The FE models of the MA TKA and KA TKA are shown in - Fig. 1. The prosthesis Genesis II Total Knee System (Smith \& Nephew Inc., Memphis, TN) was used for both MA TKA and KA TKA in CR and PS TKA.

The FE investigation model was used for stance phase gait and deep knee bend loadings. The loading condition was applied to evaluate the effect of MA TKA and KA TKA on the TKA model; the stance phase gait cycle and deep knee bend loading conditions were applied to both the tibiofemoral and patellofemoral joint motions. ${ }^{29,30}$ In addition, these loading conditions were applied to both CR and PS TKA to evaluate the biomechanical effects of KATKA and MATKA on different types of TKA. The FE model was analyzed using ABAQUS software (version 6.11; Simulia, Providence, RI). The results for the MCL and LCL forces were evaluated, and the maximum contact stress on the PE insert and the patellar button pressure were evaluated in both the MA TKA and KA TKA surgical techniques.

\section{Results}

\section{Effects of MA TKA and KA TKA on the Collateral Ligament Forces}

- Figure 2 shows the ligament forces on the MCL, LCL, popliteofibular ligament (PFL), and anterior lateral ligament (ALL) for the MA and KA in both CR and PS TKA FE models during the stance phase gait cycle. The ligament forces on the MCL were 63 and 41\% lower in CR and PS KA TKA than in CR and PS MA TKA, respectively, during the stance phase gait cycle condition. However, there was no remarkable difference in the LCL, PFL, and ALL between the two surgical alignment techniques under a stance phase gait cycle.

-Figure 3 shows the ligament forces on the MCL, LCL, PFL, and ALL for the MA and KA in both CR and PS TKA FE models in the deep knee bend loading condition are shown in -Fig. 4. The ligament forces on the MCL were 69 and 52\% lower in CR and PS KA TKA than CR and PS MA TKA, respectively, in the deep knee bend condition. The ligament forces on the ALL and PFL were greater by 18 and 23\%, and by 23 and 29\%, respectively, in CR and PS KA TKA and CR and PS MA TKA in deep knee bend conditions.

\section{Effects of MA TKA and KA TKA on the Maximum} Contact Stress on the Medial PE Insert in CR and PS TKA

- Figure 5 shows the maximum contact stress on the medial PE inserts in the MA TKA and KA TKA FE models during the stance phase gait and deep knee bend loading conditions in CR and PS TKA. The maximum contact stresses were 32 and $18 \%$ lower in CR and PS KATKA on the medial PE insert than in CR and PS MA TKA, respectively, in the stance phase gait loading condition. 

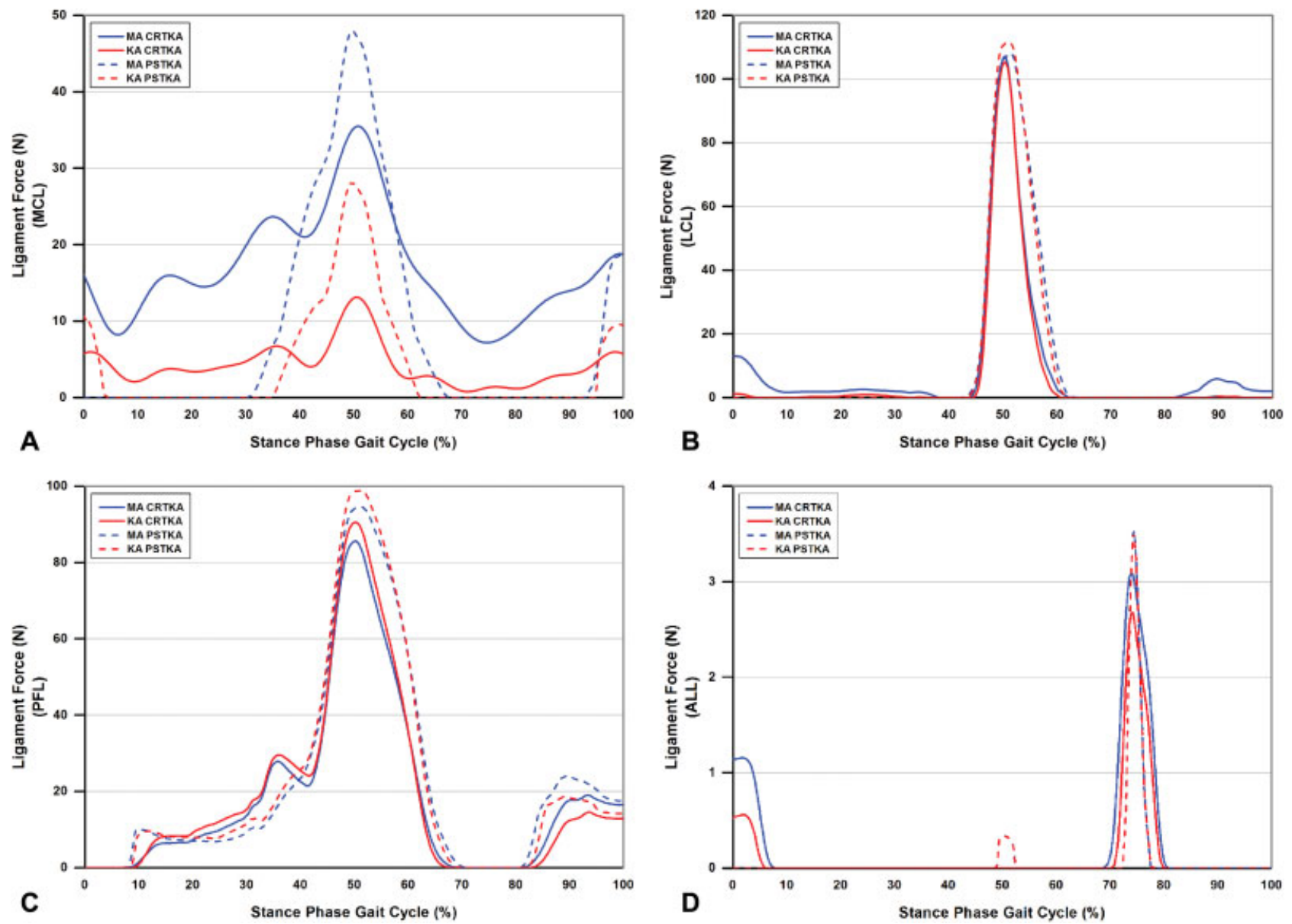

Fig. 2 Ligament forces on the (A) medial collateral ligament (MC)L, (B) lateral collateral ligament (LCL), (C) popliteofibular ligament (PFL), and (D) anterior lateral ligament (ALL) during the stance phase gait loading condition. Abbreviations: KA, kinematic alignment; MA, mechanical alignment; TKA, total knee arthroplasty.

The reduction in the maximum contact stress for the medial PE insert was better shown at deep knee bend loading conditions. The maximum contact stresses on the medial PE insert were 43 and $21 \%$ lower in CR and PS KA TKA compared with CR and PS MATKA, respectively, in the deep knee bend loading condition.

\section{Effects of MA TKA and KA TKA on the Maximum Contact Stress on the Lateral PE Insert in CR and PS TKA} The maximum contact stress on the lateral PE inserts in the MA TKA and KA TKA FE models during the stance phase gait and deep knee bend loading condition in CR and PS TKA are shown in - Fig. 6 . There was an opposite trend on the lateral side in PS TKA during the stance phase gait loading condition. The maximum contact stress was $8 \%$ greater in PS KA TKA on the lateral PE insert than in PS MA TKA during the stance phase gait loading condition. However, the contact stress on the lateral PE insert in CR KA TKA was lower than that in CR MA TKA during the stance phase gait loading condition. During deep knee bend condition, the maximum contact stress on the lateral PE insert was greater in CR and PS KA TKA than those in CR and PS MATKA, unlike the stance phase gait loading condition. - Figure 7 shows the maximum contact stress distribution on the PE inserts in the FE model during stance phase gait and deep knee bend loading conditions.

\section{Effects of MA TKA and KA TKA on the Maximum} Contact Stress on the Patellar Button in CR and PS TKA The maximum contact stresses on the patellar button in the FE model are shown in - Fig. $\mathbf{8}$ in stance phase gait and deep knee bend conditions. In both MA TKA and KA TKA, there was a negligible difference of less than $3 \%$ during the stance phase gait and deep knee bend conditions. - Figure 4 shows the maximum contact stress distribution on the patellar button in the FE model during stance phase gait and deep knee bend loading conditions.

\section{Discussion}

The most important findings of the study were that KA resulted in less collateral ligament force and lower tibial insert contact stress than MA for both the CR and PS knee without changing the contact stress in the patellofemoral compartments.

Theoretically, proper alignment is intended to minimize stress on the implant and not overload the collateral ligaments, and this should be effective during weight-bearing activities such as the stance phase of gait and deep knee loading. Inappropriate joint alignment can lead to increased stress on the prosthesis, unexpected patient outcomes, and 

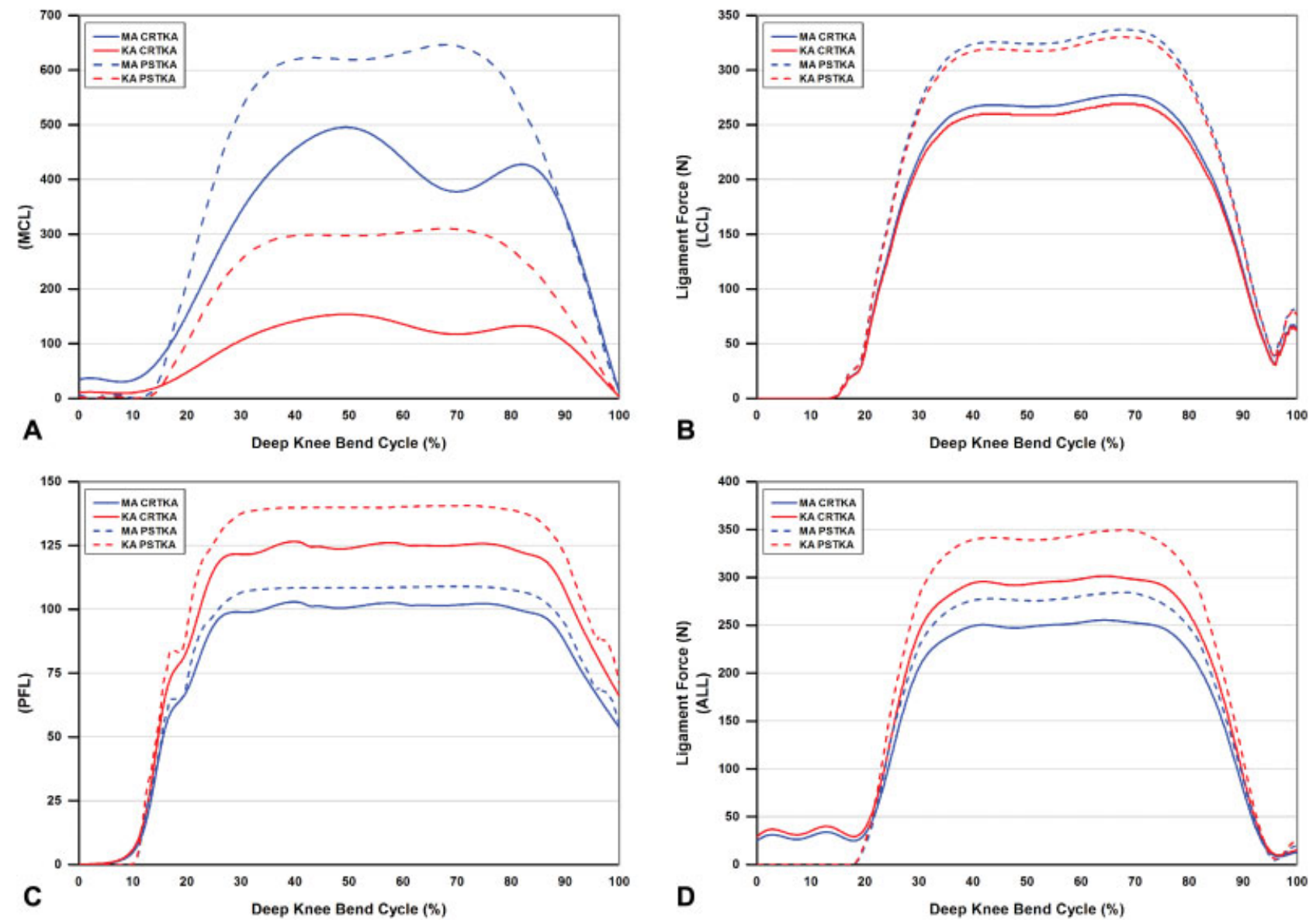

Fig. 3 Ligament forces on the (A) medial collateral ligament (MCL), (B) lateral collateral ligament (LCL), (C) popliteofibular ligament (PFL), and (D) anterior lateral ligament (ALL) during the deep knee bend loading condition. Abbreviations: KA, kinematic alignment; MA, mechanical alignment; TKA, total knee arthroplasty.

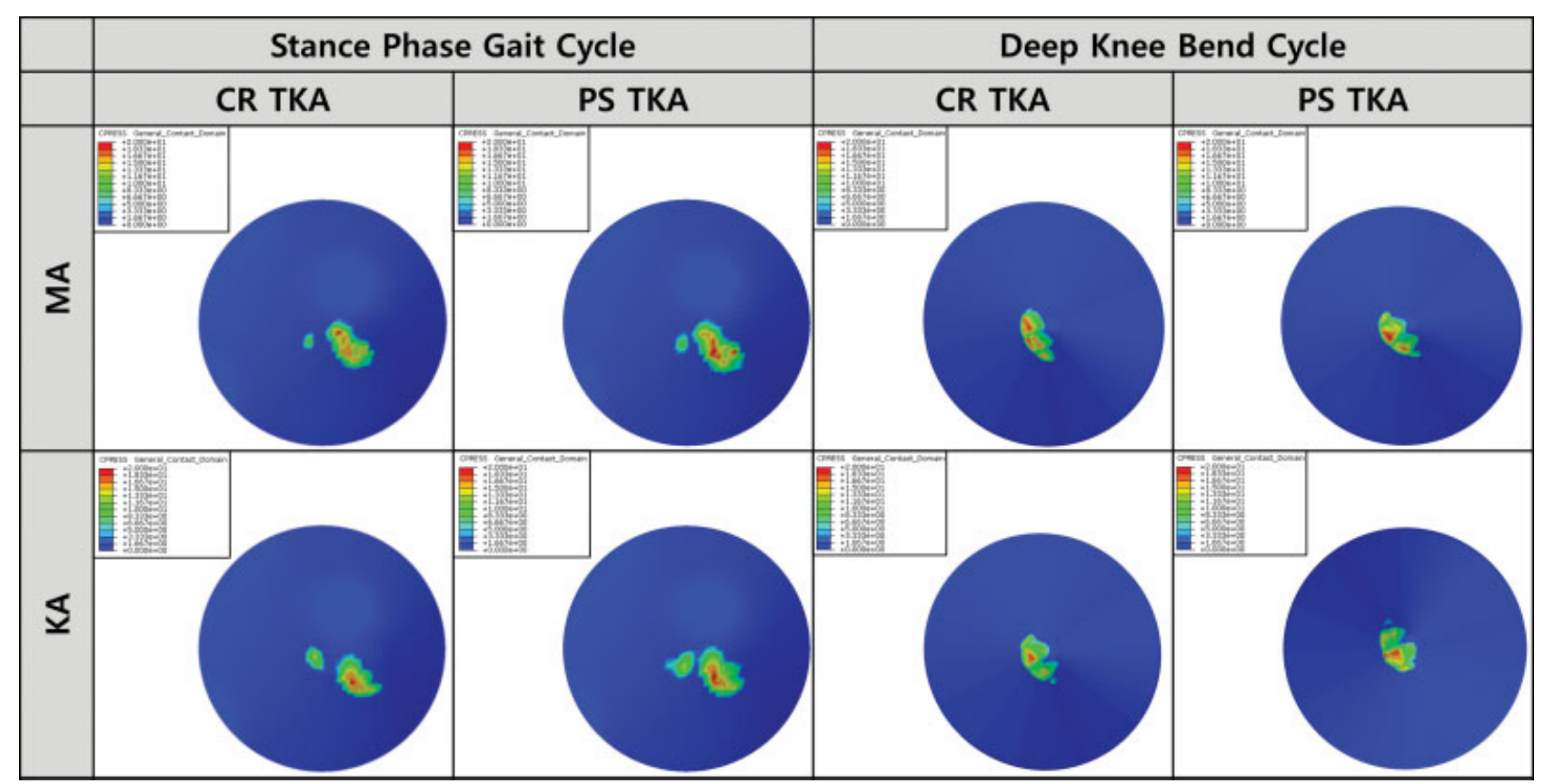

Fig. 4 Contact stress distributions on the patellar button with respect to mechanical alignment (MA) and kinematic alignment (KA) for cruciate retaining (CR) total knee arthroplasty (TKA) and posterior stabilized (PS) TKA during the stance phase gait and deep knee bend loading condition. 

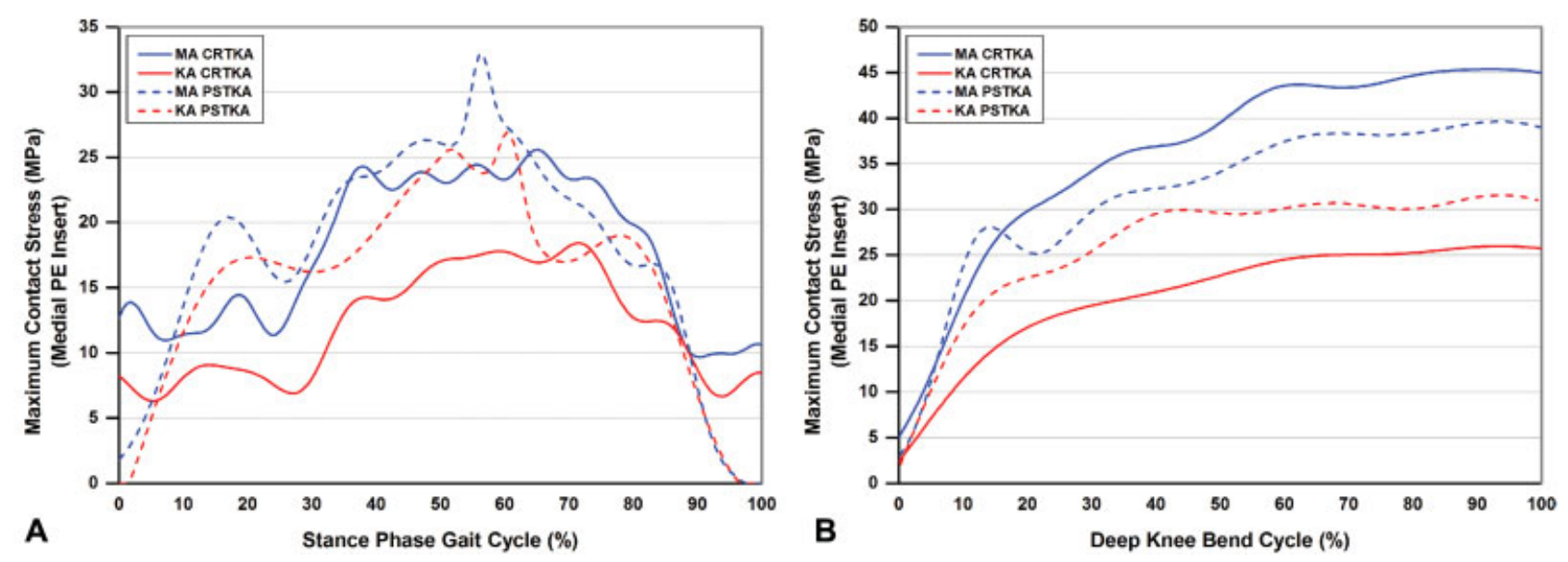

Fig. 5 Maximum contact stress on the medial polyethylene (PE) inserts in the mechanical alignment (MA) total knee arthroplasty (TKA) and kinematic alignment (KA) TKA finite element (FE) models of cruciate retaining (CR) and posterior stabilized (PS) TKA during the (A) stance phase gait and (B) deep knee bend loading conditions.
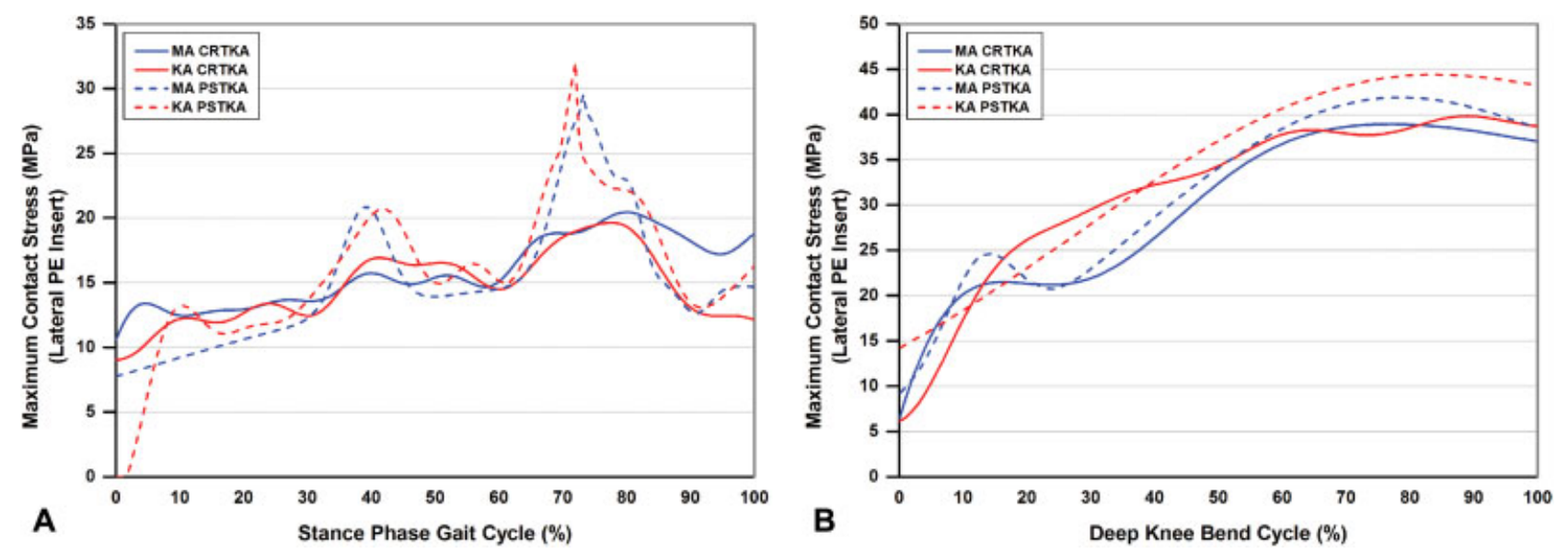

Fig. 6 Maximum contact stress on the lateral polyethylene (PE) inserts in the mechanical alignment (MA) total knee arthroplasty (TKA) and kinematic alignment (KA) TKA finite element (FE) models of cruciate retaining (CR) and posterior stabilized (PS) TKA during the (A) stance phase gait and (B) deep knee bend loading conditions.

reduction in survivorship. ${ }^{5}$ Historically, the goal of TKA has been accepted to be the restoration of joint alignment to within $3^{\circ}$ from the mechanical axis; however, recent reports have challenged the theory that outliers may result in an increased rate of revision surgery. ${ }^{5}$ Howell et al introduced KA in an attempt to address the high rates of patient dissatisfaction after TKA and the wide variety of involved preoperative alignments. ${ }^{3,14,19,21,31}$ This method considers the individualization of alignment in the human knee joint by using medical imaging and computer software to preserve prearthritic lower-extremity alignment through the restoration of the axes of rotation. Howell et al reported 214 KA CR TKAs at a mean of 31 months postoperative follow-up in three groups: limbs in the neutral range $\left(0^{\circ}\right.$, standard deviation $\left.3^{\circ}\right)$, varus alignment $\left(>3^{\circ}\right)$, and valgus alignment $\left(>3^{\circ}\right) .{ }^{21}$ They concluded that a high risk for severe failure was not observed with KA TKA in the short term and that it could be beneficial to surgeons committed to cutting the tibia perpendicular to its mechanical axis. ${ }^{21}$ In addition, Innocenti et al reported that malalignment in the tibial component was always associated with more detrimental effects compared with the femoral component under vertical loading using computational simulation. ${ }^{32}$ However, Nedopil et al showed that KA TKA restored native left to right symmetry of the hip-knee-ankle angle, distal lateral femoral angle, and proximal medial tibial angle in nearly all patients with negligible risk of varus alignment of the tibial component with respect to the native tibial joint line. ${ }^{33}$ In addition, Shelton et al showed that KA TKA has a high proportion of varus or valgus outliers using mechanically aligned criteria; the intraoperative forces in the medial and lateral compartments of patients with outlier alignment were comparable with those with in-range alignment, with no evidence of overload of the medial or lateral compartment of the knee. ${ }^{34}$ However, there has been no study that evaluated the biomechanical effects of MATKA and KA TKA with respect to the contact stresses on the PE insert and patellar button and the forces exerted on the collateral ligaments. Furthermore, there has been no study that evaluated the difference in biomechanical effect of KA TKA between CR and PS TKA. For this reason, we evaluated these parameters in the medial lateral tibiofemoral and patellofemoral joint of CR and PS 


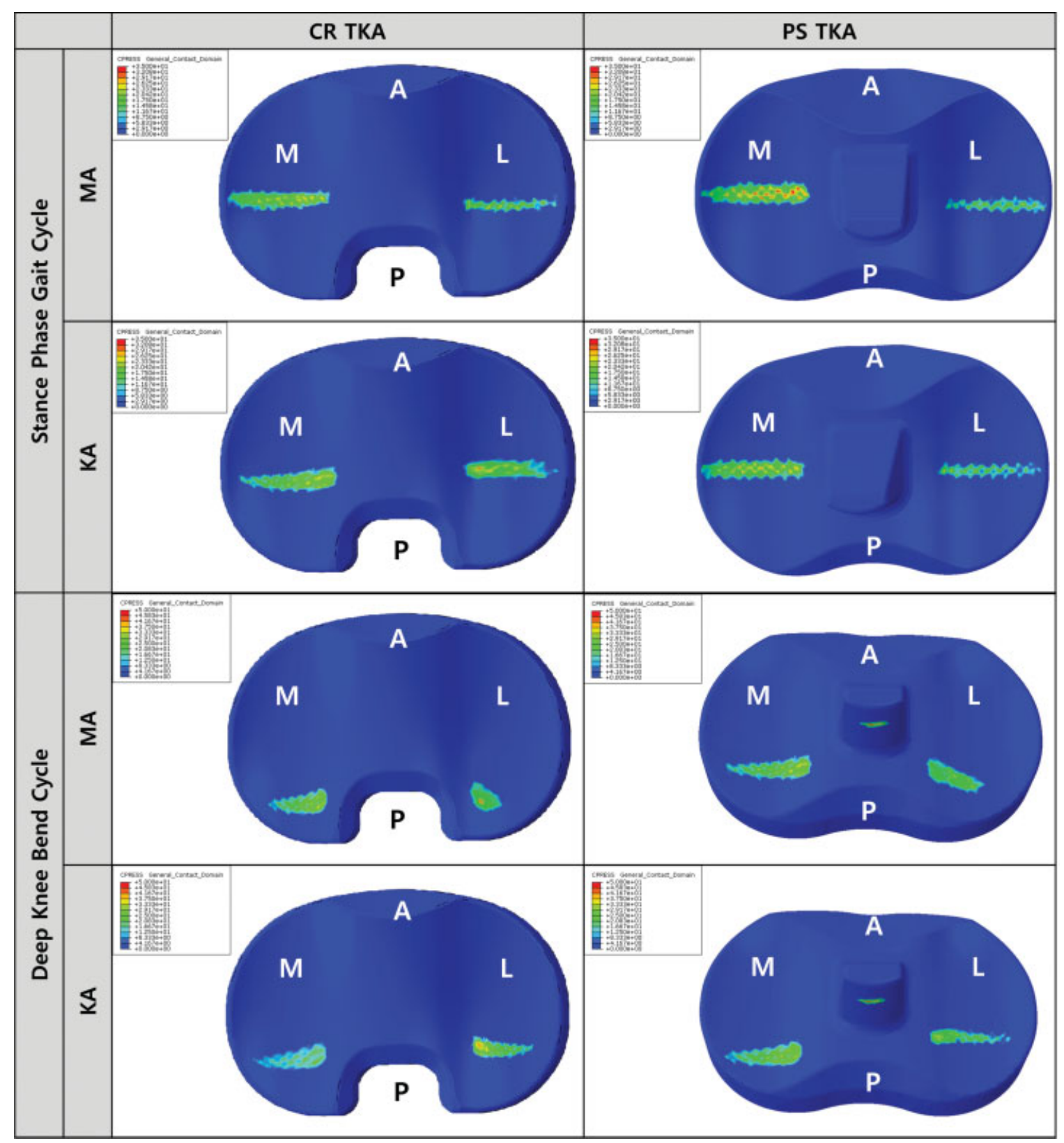

Fig. 7 Contact stress distributions on the polyethylene (PE) inserts with respect to mechanical alignment (MA) and kinematic alignment (KA) for cruciate retaining (CR) total knee arthroplasty (TKA) and posterior stabilized (PS) TKA during the stance phase gait and deep knee bend loading condition.

TKA placed with KA and MA during stance phase gait and deep knee bend loading conditions. The advantage of a computational simulation using a single subject is that we could determine the effects of component alignment within the same subject without the effect of variables such as weight, height, bony geometry, ligament properties, and component size. ${ }^{35}$

Our results showed that contact stress on the medial PE insert in KA TKA was lower than in MA TKA during the stance-phase gait and deep knee bend loading condition. Previous biomechanical studies have reported that the contact stress on the medial side is higher than that on the lateral side after TKA surgery. ${ }^{36-39}$ This trend is also seen in our MA TKA model. However, one interesting finding is that the contact stress of the medial side is lowered in KA TKA. A previous knee simulation study suggests that under static loading, a tibial malposition of $3^{\circ}$ or more in varus or valgus can greatly alter the distribution of pressure and the load between the medial and lateral compartments. ${ }^{38}$ We demonstrated this effect by changing from MA to KA. The findings of the current study agree with those of the previous biomechanical study. ${ }^{40}$

There were no improvements in the biomechanical effects on the contact stress on the lateral PE insert in KA TKA compared with MA TKA in the stance-phase gait and deep knee-bend loading conditions. An interesting finding was that the maximum contact stress on the lateral side was greater in CR MA TKA compared with CR KA TKA. This means that CR KA 

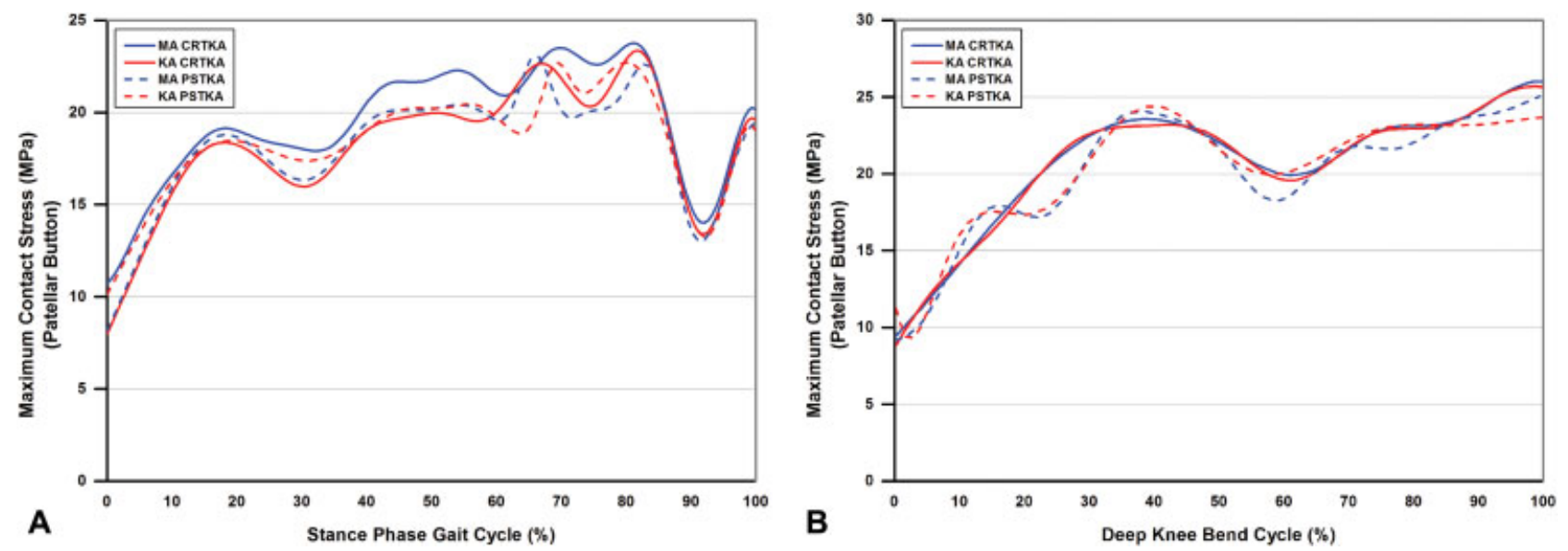

Fig. 8 Maximum contact stresses on the patellar button for mechanical alignment (MA) and kinematic alignment (KA) in cruciate retaining (CR) total knee arthroplasty (TKA) and posterior stabilized (PS) TKA during the (A) stance phase gait and (B) deep knee bend loading conditions.

TKA provides a better biomechanical effect on the lifespan of the prosthesis wear and knee-joint instability than CR MATKA. Our results show that CR KA TKA provided a better biomechanical effect on the knee joint than did PS KA TKA.

The purpose of KATKA is to correct the arthritic deformity of the lower extremity to the normal prearthritic alignment with the alignment of rotational axes in the femoral and tibial components, by means of setting the anterior-posterior axis of the tibial component parallel to the flexionextension tibial reference line, which can restore the natural tibiofemoral articular surfaces, alignment, and laxities of the knee joint. ${ }^{3,17,41}$ It is fundamental to minimize the external rotation of the tibial component with respect to the femoral component in knee flexion, as it affects the patellofemoral tracking and increases the contact forces on the patellar button, leading to patella failure. ${ }^{42}$ However, there was no difference in the contact stresses on the patellar button between MA TKA and KA TKA without malrotation in neutral knee alignment.

Another interesting finding is that the force exerted on the MCL was lower in KA TKA than in MA TKA. In a previous study, it was reported that TKA using MA required the management of complex and uncorrectable collateral ligament imbalance, and it led to a wide range of changes in tibiofemoral alignment from normal knee conditions. ${ }^{43,44}$ KATKA has the advantage of preventing imbalance of the collateral ligaments, which have a wide variety of complex mechanisms that are not correctable through collateral ligament release, as well as a wide range of changes in the natural alignment of the lower extremity resulting from MA TKA. KA TKA restores the normal alignment of the lower extremity and the joint line of the knee, thus providing more physiological forces exerted on the collateral ligaments than MA TKA, which is unnaturally aligned to a neutral MA. ${ }^{45}$ Our results showed that the forces exerted on the MCL in PS and CR KA TKA were 41 and 53\% less than those in MATKA in the stance phase gait loading condition. Similarly, they showed that the forces exerted on the MCL in PS and CR KATKA were 63 and 69\% less than those in MATKA in the deep knee bend loading condition.
The tightened MCL might help indicate the reported unsatisfactory pain in clinical observations. ${ }^{46}$ Such an imbalanced soft-tissue loading resulted in changes in the predicted knee contact stress, especially as the knee moved from flexion into extension. Our study demonstrated that the contact stress of the medial PE insert was reduced by reducing the force on the MCL. In addition, the result that the forces exerted on ligaments in CR TKA were always lower than those in PS TKA indicates good agreement with previous studies. ${ }^{35}$ The contact stress and ligament force also decreased more in CR KA TKA than in PS KA TKA. We found that the biomechanical effects of KA and MA TKA are different. Based on our results, KA is more effective in CR TKA.

This study has several limitations. (1) Only the intact model was validated. However, FE analysis with validated FE models has been widely used in orthopedic biomechanics for the assessment of surgical technique or evaluation of implant longevity. ${ }^{24,25,47-49}$ We developed the computational model by using data from only a young, male subject. Analyzing subjects of various ages would improve the validity of the results, because the validity is also dependent on the geometry of the knee joint. Furthermore, we eliminated other biomechanical factors by using a young male subject without a history of knee injury or patellar subluxation. (2) The balance of all the collateral ligaments was accurate in our FE model because other biomechanical effects would need to be considered otherwise. Moreover, KA TKA is the strategy that strives to restore prearthritic knee alignment and avoid the release of the collateral ligament. ${ }^{17,31,41}$ (3) The results could not substitute clinical results and patient satisfaction, as they represent outcomes from FE analysis. However, contact stress on the PE insert and force exerted on ligaments are key factors that should be investigated for the evaluation of biomechanical effects in computational biomechanics. ${ }^{23,35,47,48,50}$ (4) Finally, only the stance-phase gait and deep knee-bend simulations were performed, and simulations involving more demanding activities, such as chair rising/sitting or climbing/descending, would be required for a more reliable investigation in the future. 
In conclusion, KA TKA provides lower maximum contact stresses on the medial PE insert and lower average contact stress, as well as lower force exerted on the MCL than provided by MATKA. In the current study, the biomechanical effect was superior in KA TKA than in MA TKA, and KA was more effective in CR TKA; however, clinical outcomes, including long-term survivorship, would also be needed when evaluating the advantages of KA TKA.

\section{Conflict of Interest}

None declared.

\section{References}

1 Maderbacher G, Schaumburger J, Keshmiri A, et al. Pinless navigation in total knee arthroplasty: navigation reduced by the maximum? Int Orthop 2015;39(03):455-460

2 Iorio R, Mazza D, Drogo P, et al. Clinical and radiographic outcomes of an accelerometer-based system for the tibial resection in total knee arthroplasty. Int Orthop 2015;39(03):461-466

3 Howell SM, Papadopoulos S, Kuznik K, Ghaly LR, Hull ML. Does varus alignment adversely affect implant survival and function six years after kinematically aligned total knee arthroplasty? Int Orthop 2015;39(11):2117-2124

4 D'Lima DD, Chen PC, Colwell CW Jr. Polyethylene contact stresses, articular congruity, and knee alignment. Clin Orthop Relat Res $2001 ;(392): 232-238$

5 Cherian JJ, Kapadia BH, Banerjee S, Jauregui JJ, Issa K, Mont MA. Mechanical, anatomical, and kinematic axis in TKA: concepts and practical applications. Curr Rev Musculoskelet Med 2014;7(02): 89-95

6 Colle F, Lopomo N, Bruni D, et al. Analysis of knee functional flexion axis in navigated TKA: identification and repeatability before and after implant positioning. Knee Surg Sports Traumatol Arthrosc 2014;22(03):694-702

7 Baker PN, van der Meulen JH, Lewsey J, Gregg PJ; National Joint Registry for England and Wales; Data from the National Joint Registry for England and Wales. The role of pain and function in determining patient satisfaction after total knee replacement J Bone Joint Surg Br 2007;89(07):893-900

8 Bourne RB, Chesworth BM, Davis AM, Mahomed NN, Charron KD. Patient satisfaction after total knee arthroplasty: who is satisfied and who is not? Clin Orthop Relat Res 2010;468(01):57-63

9 Noble PC, Conditt MA, Cook KF, Mathis KB. The John Insall Award: patient expectations affect satisfaction with total knee arthroplasty. Clin Orthop Relat Res 2006;452(452):35-43

10 Dy CJ, Marx RG, Bozic KJ, Pan TJ, Padgett DE, Lyman S. Risk factors for revision within 10 years of total knee arthroplasty. Clin Orthop Relat Res 2014;472(04):1198-1207

11 Parratte S, Pagnano MW, Trousdale RT, Berry DJ. Effect of postoperative mechanical axis alignment on the fifteen-year survival of modern, cemented total knee replacements. J Bone Joint Surg Am 2010;92(12):2143-2149

12 Bellemans J, Colyn W, Vandenneucker H, Victor J. The Chitranjan Ranawat award: is neutral mechanical alignment normal for all patients? The concept of constitutional varus. Clin Orthop Relat Res 2012;470(01):45-53

13 Park A, Duncan ST, Nunley RM, Keeney JA, Barrack RL, Nam D. Relationship of the posterior femoral axis of the "kinematically aligned" total knee arthroplasty to the posterior condylar, transepicondylar, and anteroposterior femoral axes. Knee 2014;21 (06):1120-1123

14 Howell SM, Hodapp EE, Vernace JV, Hull ML, Meade TD. Are undesirable contact kinematics minimized after kinematically aligned total knee arthroplasty? An intersurgeon analysis of consecutive patients. Knee Surg Sports Traumatol Arthrosc 2013;21(10):2281-2287

15 Dossett HG, Estrada NA, Swartz GJ, LeFevre GW, Kwasman BG. A randomised controlled trial of kinematically and mechanically aligned total knee replacements: two-year clinical results. Bone Joint J 2014;96-B(07):907-913

16 Nam D, Nunley RM, Barrack RL. Patient dissatisfaction following total knee replacement: a growing concern? Bone Joint J 2014;96B(11, Supple A):96-100

17 Brar AS, Howell SM, Hull ML. What are the bias, imprecision, and limits of agreement for finding the flexion-extension plane of the knee with five tibial reference lines? Knee 2016;23(03):406-411

18 Nam D, Lin KM, Howell SM, Hull ML. Femoral bone and cartilage wear is predictable at $0^{\circ}$ and $90^{\circ}$ in the osteoarthritic knee treated with total knee arthroplasty. Knee Surg Sports Traumatol Arthrosc 2014;22(12):2975-2981

19 Howell SM, Papadopoulos S, Kuznik KT, Hull ML. Accurate alignment and high function after kinematically aligned TKA performed with generic instruments. Knee Surg Sports Traumatol Arthrosc 2013;21(10):2271-2280

20 Nedopil AJ, Howell SM, Hull ML. What mechanisms are associated with tibial component failure after kinematically aligned TKA? Int Orthop 2017;41(08):1561-1569

21 Howell SM, Howell SJ, Kuznik KT, Cohen J, Hull ML. Does a kinematically aligned total knee arthroplasty restore function without failure regardless of alignment category? Clin Orthop Relat Res 2013;471(03):1000-1007

22 Kim YS, Kang KT, Son J, et al. Graft extrusion related to the position of allograft in lateral meniscal allograft transplantation: biomechanical comparison between parapatellar and transpatellar approaches using finite element analysis. Arthroscopy 2015;31 (12):2380-91.e2

23 Kwon OR, Kang KT, Son J, et al. Biomechanical comparison of fixed- and mobile-bearing for unicomparmental knee arthroplasty using finite element analysis. J Orthop Res 2014;32(02): 338-345

24 Peña E, Calvo B, Martinez MA, Palanca D, Doblaré M. Why lateral meniscectomy is more dangerous than medial meniscectomy. A finite element study. J Orthop Res 2006;24(05):1001-1010

25 Mesfar W, Shirazi-Adl A. Biomechanics of the knee joint in flexion under various quadriceps forces. Knee 2005;12(06):424-434

26 Takeda Y, Xerogeanes JW, Livesay GA, Fu FH, Woo SL. Biomechanical function of the human anterior cruciate ligament. Arthroscopy 1994;10(02):140-147

27 Blankevoort L, Huiskes R. Validation of a three-dimensional model of the knee. J Biomech 1996;29(07):955-961

28 Godest AC, Beaugonin M, Haug E, Taylor M, Gregson PJ. Simulation of a knee joint replacement during a gait cycle using explicit finite element analysis. J Biomech 2002;35(02):267-275

29 Baldwin MA, Clary C, Maletsky LP, Rullkoetter PJ. Verification of predicted specimen-specific natural and implanted patellofemoral kinematics during simulated deep knee bend. J Biomech 2009;42(14):2341-2348

30 Kang KT, Koh YG, Son J, et al. Measuring the effect of femoral malrotation on knee joint biomechanics for total knee arthroplasty using computational simulation. Bone Joint Res 2016;5 (11):552-559

31 Howell SM, Rogers SL. Method for quantifying patient expectations and early recovery after total knee arthroplasty. Orthopedics 2009;32(12):884

32 Innocenti B, Bellemans J, Catani F. Deviations from optimal alignment in TKA: is there a biomechanical difference between femoral or tibial component alignment? J Arthroplasty 2016;31 (01):295-301

33 Nedopil AJ, Singh AK, Howell SM, Hull ML. Does calipered kinematically aligned TKA restore native left to right symmetry of the lower limb and improve function? J Arthroplasty 2018;33(02): 398-406 
34 Shelton TJ, Nedopil AJ, Howell SM, Hull ML. Do varus or valgus outliers have higher forces in the medial or lateral compartments than those which are in-range after a kinematically aligned total knee arthroplasty? Limb and joint line alignment after kinematically aligned total knee arthroplasty. Bone Joint J 2017;99-B(10):1319-1328

35 Thompson JA, Hast MW, Granger JF, Piazza SJ, Siston RA. Biomechanical effects of total knee arthroplasty component malrotation: a computational simulation. J Orthop Res 2011;29(07):969-975

36 D'Lima DD, Steklov N, Fregly BJ, Banks SA, Colwell CW Jr. In vivo contact stresses during activities of daily living after knee arthroplasty. J Orthop Res 2008;26(12):1549-1555

37 Chen Z, Wang L, Liu Y, et al. Effect of component mal-rotation on knee loading in total knee arthroplasty using multi-body dynamics modeling under a simulated walking gait. J Orthop Res 2015;33(09):1287-1296

38 Werner FW, Ayers DC, Maletsky LP, Rullkoetter PJ. The effect of valgus/varus malalignment on load distribution in total knee replacements. J Biomech 2005;38(02):349-355

39 Zhao D, Banks SA, D'Lima DD, Colwell CW Jr, Fregly BJ. In vivo medial and lateral tibial loads during dynamic and high flexion activities. J Orthop Res 2007;25(05):593-602

40 Nakamura S, Tian Y, Tanaka Y, et al. The effects of kinematically aligned total knee arthroplasty on stress at the medial tibia: a case study for varus knee. Bone Joint Res 2017;6(01):43-51

41 Howell SM, Hull ML. Principles of Kinematic Alignment in Total Knee Arthroplasty with and without Patient Specific Cutting Blocks. Elsevier; 2012:1255-1268. Doi: 10.1016/B978-1-43771503-3.00120-7

42 Bellemans J, Banks S, Victor J, Vandenneucker H, Moemans A. Fluoroscopic analysis of the kinematics of deep flexion in total knee arthroplasty. Influence of posterior condylar offset. J Bone Joint Surg Br 2002;84(01):50-53
43 Gu Y, Howell SM, Hull ML. Simulation of total knee arthroplasty in $5^{\circ}$ or $7^{\circ}$ valgus: a study of gap imbalances and changes in limb and knee alignments from native. J Orthop Res 2017;35(09): 2031-2039

$44 \mathrm{Gu} \mathrm{Y,} \mathrm{Roth} \mathrm{JD,} \mathrm{Howell} \mathrm{SM,} \mathrm{Hull} \mathrm{ML.} \mathrm{How} \mathrm{frequently} \mathrm{do} \mathrm{four}$ methods for mechanically aligning a total knee arthroplasty cause collateral ligament imbalance and change alignment from normal in white patients? AAOS Exhibit Selection. J Bone Joint Surg Am 2014;96(12):e101

45 Delport H, Labey L, Innocenti B, De Corte R, Vander Sloten J, Bellemans J. Restoration of constitutional alignment in TKA leads to more physiological strains in the collateral ligaments. Knee Surg Sports Traumatol Arthrosc 2015;23(08): 2159-2169

46 Bell SW, Young P, Drury C, et al. Component rotational alignment in unexplained painful primary total knee arthroplasty. Knee 2014;21(01):272-277

47 Perillo-Marcone A, Taylor M. Effect of varus/valgus malalignment on bone strains in the proximal tibia after TKR: an explicit finite element study. J Biomech Eng 2007;129(01):1-11

48 Liau JJ, Cheng CK, Huang CH, Lo WH. The effect of malalignment on stresses in polyethylene component of total knee prostheses-a finite element analysis. Clin Biomech (Bristol, Avon) 2002;17(02): 140-146

49 Kim HJ, Kang KT, Son J, Lee CK, Chang BS, Yeom JS. The influence of facet joint orientation and tropism on the stress at the adjacent segment after lumbar fusion surgery: a biomechanical analysis. Spine J 2015;15(08):1841-1847

50 Kang KT, Koh YG, Son J, et al. Measuring the effect of femoral malrotation on knee joint biomechanics for total knee arthroplasty using computational simulation. Bone Joint Res 2016;5 (11):552-559 